\title{
Good Recovery of Prostate Carcinoma with Spine Metastases to T9 Following Laminectomy
}

\author{
Muhammad Wafiuddin', ${ }^{1}$, Ed Simor Khan ${ }^{2 *}$, Ahmad Faizal ${ }^{1}$, Jasfizal Jasnï ${ }^{3}$, Loh Li Loong²
}

${ }^{1}$ Surgical-Based Department,

Faculty of Medicine and Health Sciences,

Universiti Malaysia Sabah, Kota Kinabalu,

Sabah, Malaysia

2 Orthopaedic and Rehabilitation Department,

Kuliyyah of Medicine, International Islamic

University Malaysia, Gombak, Selangor,

Malaysia

${ }^{3}$ Orthopaedic Department,

National Defence University of Malaysia,

Kuala Lumpur, Malaysia

\section{*Corresponding author's email: \\ drwafi1986@gmail.com}

Received: 14 November 2018

Accepted: 7 October 2019

Keywords:

prostate carcinoma metastasis,

laminectomy, Frankel Grade A

\section{ABSTRACT}

Prostate carcinoma is a common health issue that can metastasise in the spine. A 65-year-old male was diagnosed with prostate carcinoma and two years later he developed a progressive neurological deficit over the bilateral lower limb. He experienced severe back pain, became paraplegic and the quality of life was severely impaired. Radiographic investigations were done and revealed osteoblastic bone metastasis at thoracic vertebrae with spinal cord compression. The patient underwent surgical decompression surgery at the T9 level mainly for pain control. Six months post-surgery not only the pain was well controlled but patient able to ambulate with walking aid. It is a rare post-operative result as the neurological recovery in a patient with complete paralysis is less than $\mathbf{3} \%$. This type of recovery is possible when the cause of the neurological deficit is mainly mechanical compression from tumour rather than cord ischaemia from traumatic injury.

\section{INTRODUCTION}

Prostate carcinoma is one of the most common problems in developed countries, $80 \%$ of patient will develop spinal metastases and $6.7 \%$ complicated with spinal compression. Back pain, weakness, anal and urinary sphincter dysfunction will lead to a decrease in quality of life ${ }^{1}$. Their median overall survival rate is 2 years. The recovery rate of a patient with a complete neurological deficit of lower limb recovered is only around $3 \%^{2}$. Usually, a patient is classified using the Frankel Classification $^{3}$ either the patient is unable to 
walk (Frankel A - C) and Frankel D and E where the patient can walk. Spinal decompression surgery is aimed to relive the pressure compressing the spinal cord by removing either the lamina, ligament, enlarging the foramina or part of the intervertebral disc. Six months after post-surgery if patient gained improvement in the neurological function he or she is further classified according to the Frankel classification. This was a case of advanced prostate cancer where good neurological recovery was achieved following laminectomy of T9 vertebra in a patient with spinal metastases.

\section{CASE PRESENTATION}

A 65-year-old male diagnosed with prostate carcinoma 2 years prior to presentation. Initially presented with nocturia and dysuria he was treated for benign prostatic hyperplasia. As time passes by he develops haematuria with significant loss of weight and appetite. He underwent orchidectomy and completed chemotherapy. Seven months later he presented with progressive weakness and numbness of bilateral lower limb weakness associated with back pain. He described the pain as stabbing in nature and located at a lower back area with radiation to the buttock. Bowel and urinary function are impaired. As the disease progress, the patient became bedbound and his quality of life worsens. Visual Analog Score for his back pain was 5/10. General examination showed a moderate build man, he was not pale and no lymph node was palpable. There was local tenderness over the thoracic and lumbar area however there was no deformity. Perianal sensation, deep anal sensation and sphincter function were normal. Neurological examination revealed complete paralysis of the bilateral lower limb, with absent of bilateral knee and ankle jerk reflex and sensation. Plain radiograph and magnetic resonance imaging (MRI) showed osteoblastic bony metastases at the T9 level with the epidural extension (Figures 1, 2, 3, and 4). The diagnosis was made at this point.

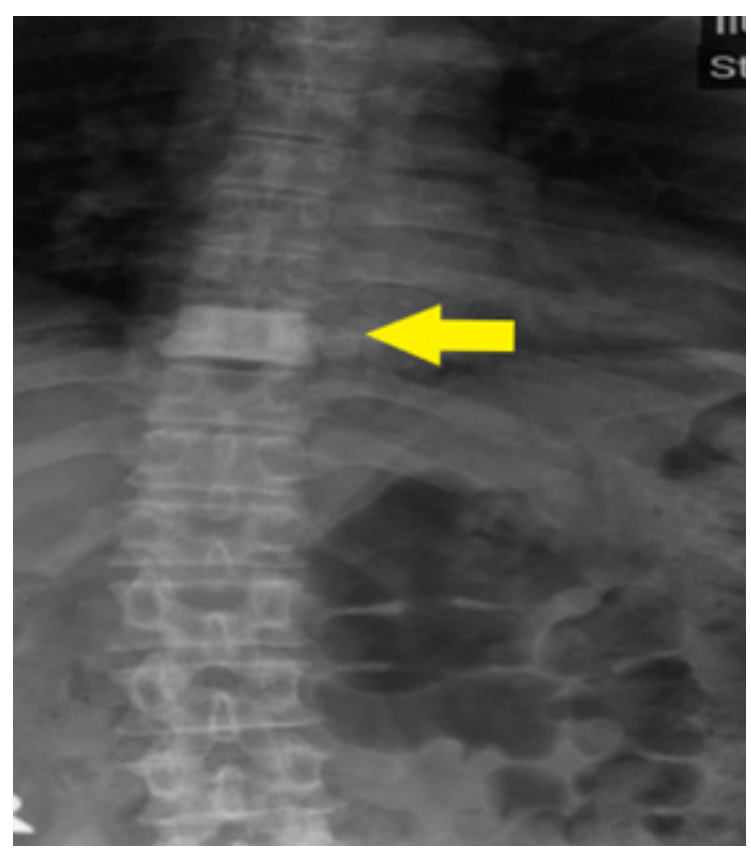

Figure 1 Posterior-anterior plain radiograph showing osteoblastic lesion (yellow arrow) over the T9 thoracic vertebrae

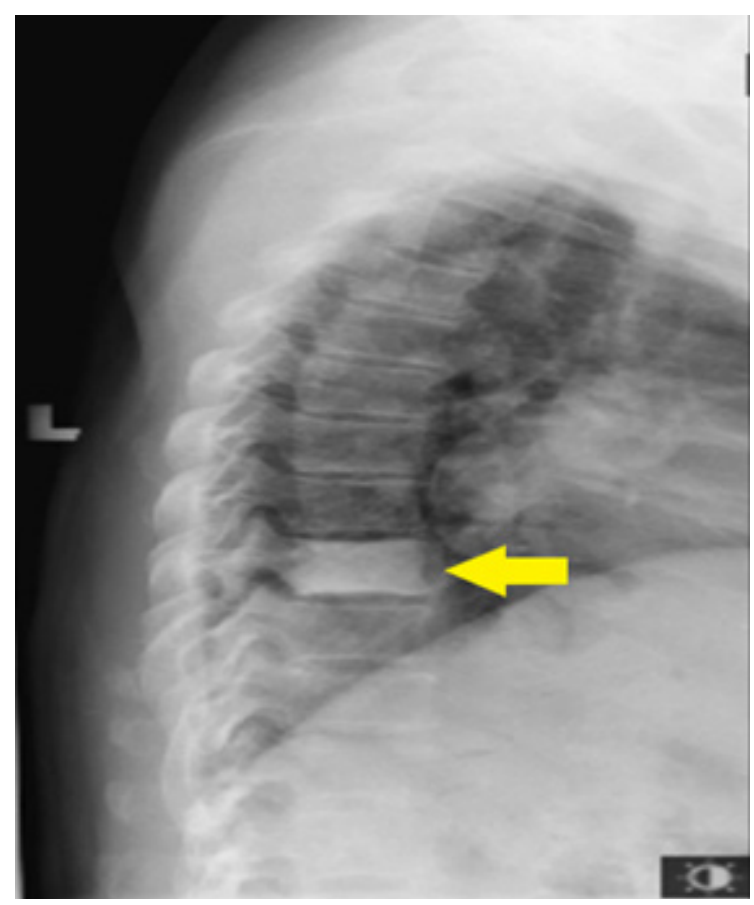

Figure 2 Lateral plain radiograph with the arrow pointing towards osteoblastic lesion over the T9 thoracic vertebrae, there is no obvious kyphosis noted 


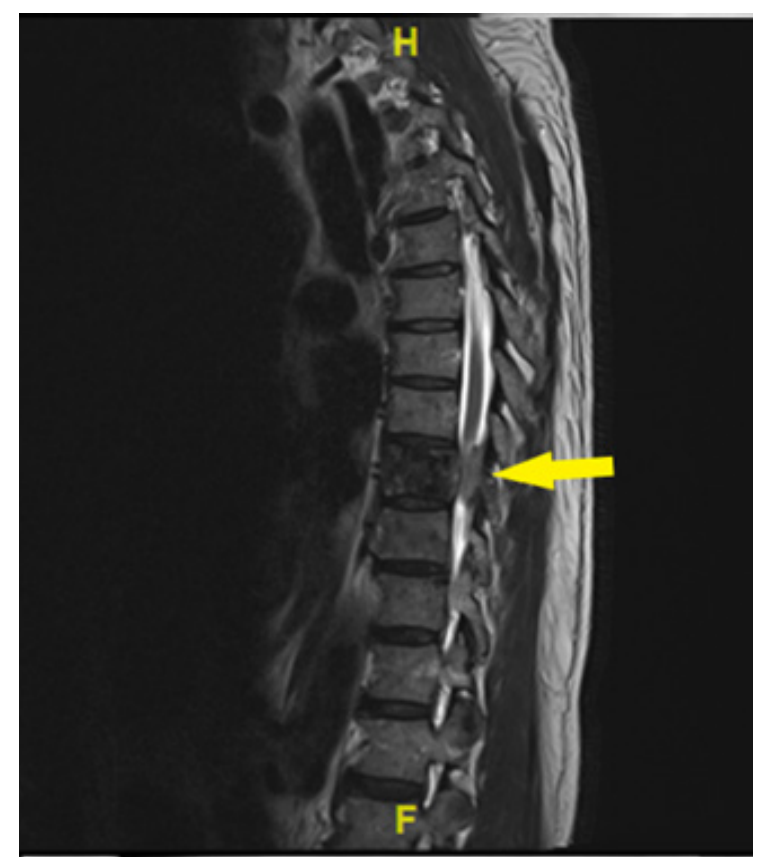

Figure $3 \mathrm{MRI}$ in the sagittal plane with Osteoblastic bone metastases at T9 level with the arrow pointing towards the epidural extension

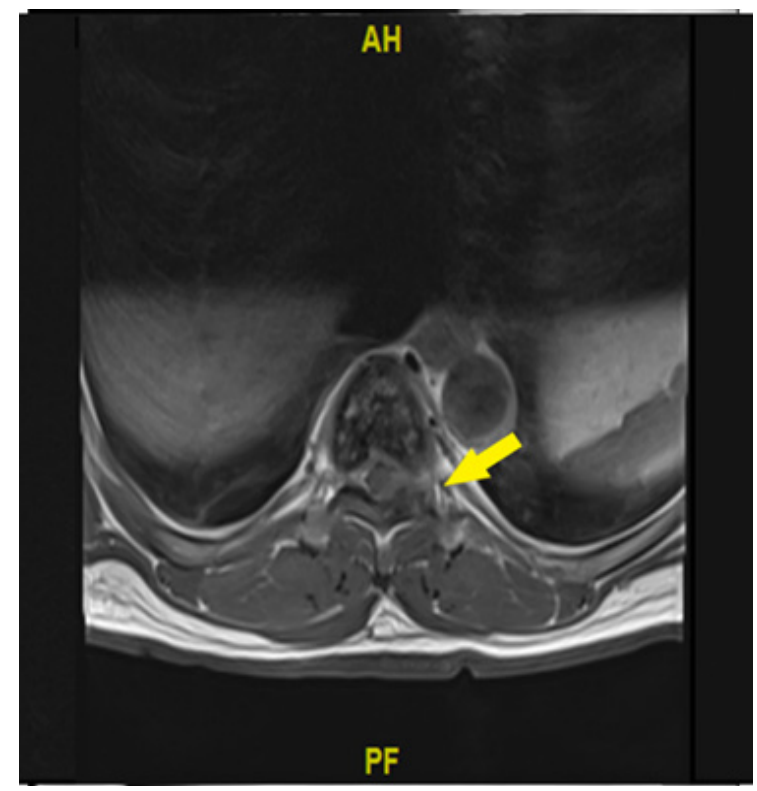

Figure 4 Axial plane MRI cut at T9 level showed osteoblastic bone metastases involving mainly the vertebrae body, with the arrow pointing towards the area where the spinal cord is compressed within the spinal canal
To predict the patient prognosis, we scored the patient based on the revised Tokuhashi score for spinal metastases ${ }^{4}$. The score was calculated taking into account of his general condition which is good during the presentation, no extraspinal metastatic foci, only one number of metastasis to the vertebral body, no metastasis to other organs, non-aggressive type of cancer, and complete paralysis. His total score was less than eight. A score of less than eight predicted that the life expectancy for this patient is less than six months. Still clinical in the dilemma we offered the patient surgery mainly for pain relief. $\mathrm{He}$ underwent wide laminectomy of T9 vertebra with preservation of bilateral facet joint. No implant needed as there is no significant vertebral body collapse. Six months after surgery, the patient showed good neurological recovery with motor power MRC grade 4 (Figures 5 and 6) for bilateral lower limb and Visual Analog Score for back pain reduced significantly. The urinary and bowel function slowly improving over time.

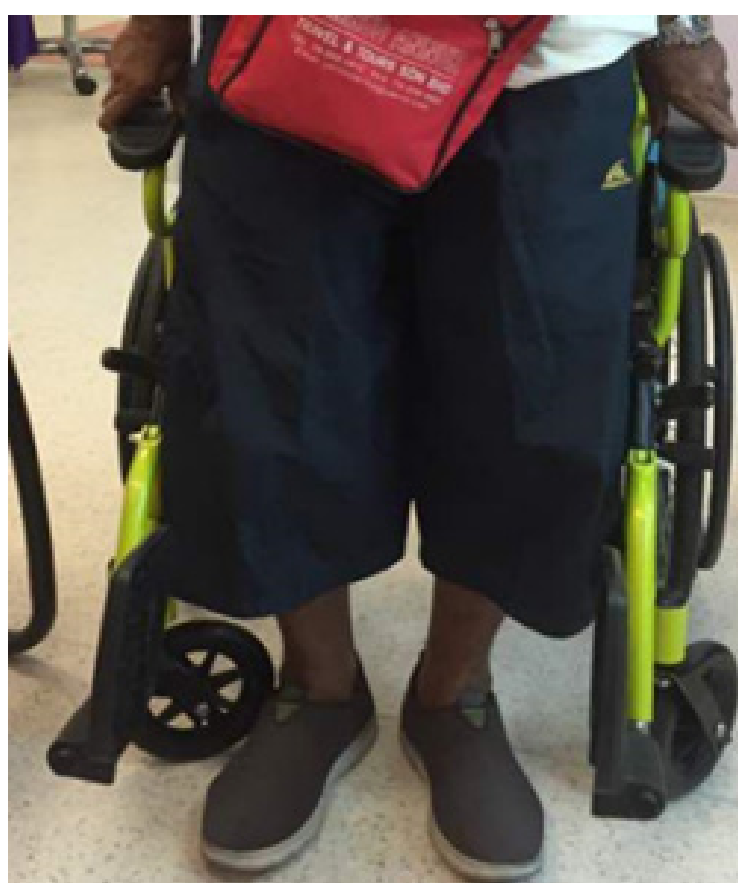

Figure 5 Six months after decompressive laminectomy patient can stand erect with MRC power grading at least 4 


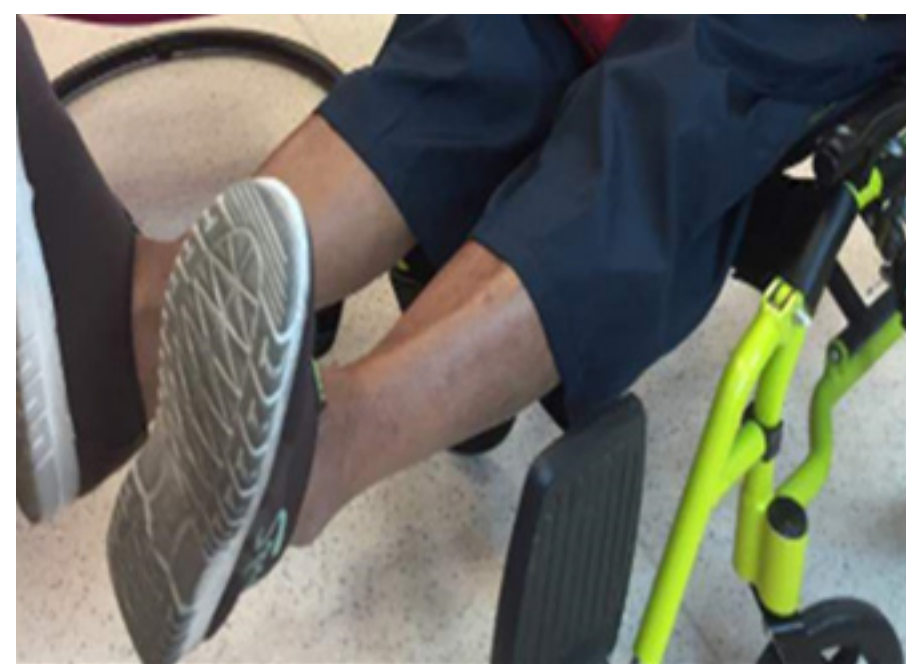

Figure 6 The patient quadriceps muscle power of at least 4 during the examination

\section{DISCUSSION}

Prostate cancer is common cancer with 1.6 million men diagnosed worldwide ${ }^{5}$. Spinal cord compression due to prostate metastases to occur at the thoracic level is the highest with an incidence of $15.7 \%{ }^{1}$. Bone metastasis is a debilitating condition and metastasis to the spine can cause cord compression, impaired bowel and urinary function and pain. Clinical management of spinal metastasis patient requires a multi-disciplinary team that includes spine surgeon's, radiation oncologist, medical oncologist rehabilitation team and supporting nurses ${ }^{6}$. In an end-stage cancer patient, the aim is to prolong life expectancy, local tumour control and to improve the overall quality of life. Offering surgery is always a dilemma due to the high rate of postoperative complications ${ }^{7}$. Furthermore, he presented with complete paralysis (Frankel A) and the success rate in regaining neurological function post-operatively is only around $3 \% \%^{2,8}$.

Spine Surgeons used multiple scoring systems to predict patient prognosis and aid in the decision making process. Modified Tokuhashi and Tomita is useful to predict patient survival. Tomita score is based on the primary tumour site, the number of visceral metastases and the number of bone metastases. While the Modified Tokuhashi score considers the general condition of the patient, the number of bone metastasis outside the spine, either metastasis to major organ removable or not, and lower limb neurological function. Modified Tokuhashi had better accuracy compared to Tomita Scoring in predicting patient survival ${ }^{4}$. A lower score means the survival is poorer and vice versa.

In general, in spinal metastasis, there is two mainstay treatment, radiotherapy and surgical decompression with or without stabilization using an implant. A throughout screening is essential to avoid further morbidity to the patient. An advanced cancer patient is immunosuppressed and malnourished making them more prone to surgical site breakdown.

In an advanced cancer patient, the surgical aim is mainly palliative to reduce the pain, control local tumour extension, and to prepare the patient for radiotherapy. Surgical strategies are divided into three parts according to the Global Spine Tumour Study Group (GSTSG) ${ }^{9}$. Surgeons aim to do palliative decompression, tumour debulking and total vebrectomy, and to achieve this method aim is further divided either by removing the tumour piece by piece or en bloc removal. The method of removal will influence the tumour margin whether it is intralesional or wide margin ${ }^{10}$. For palliative surgery the tactic is palliative 
decompression, piece by piece tumour excision and margin will be intralesional.

Three to four weeks post-operative and provided the wound is healed patient can underwent adjuvant radiotherapy regime. Typically, external beam radiotherapy with a single fraction dose of $8 \mathrm{~Gy}$ is used to provide palliative pain relief. The efficacy of external beam radiotherapy in reducing pain is almost up to $60 \%{ }^{11}$.

\section{CONCLUSION}

The aim of surgery and adjuvant radiotherapy is to provide pain relief in a palliative patient. We did not expect neurological recovery after the decompressive spinal surgery. He recovered from MRC power zero to four for bilateral lower limb and regain his urinary and bowel function. As the chance of recovery is only $3 \%$, his post-operative findings are rare. There is a chance of significant neurological recovery following decompression surgery for spine metastases from prostate cancer especially when the cause of the neurological deficit is mainly mechanical compression from tumour rather than cord ischaemia from traumatic injury.

\section{ACKNOWLEDGEMENTS}

We would like to acknowledge the patient for his permission in using his clinical history and findings.

\section{CONFLICT OF INTEREST}

The authors declare that they have no competing interests in publishing this case.

\section{CONSENTS}

Written informed consent was obtained from the patient to publish the case with its related pictures. A copy of the written consent is available for review by the Chief Editor.

\section{REFERENCES}

1. Cereceda LE, Flechon A, Droz JP. (2003). Management of vertebral metastases in prostate cancer: a retrospective analysis in 119 patients. Clinical Prostate Cancer 2 (1): 34 - 40. DOI: https://doi.org/10.3816/ CGC.2003.n.010

2. Jansson KA, Bauer HC. (2006) Survival, complications and outcome in 282 patients operated for neurological deficit due to thoracic or lumbar spinal metastases. Eur Spine J 15 (2): 196 - 202. DOI: 10.1007/ s00586-004-0870-6

3. Roberts TT, Leonard GR, Cepela DJ. (2017). Classifications in brief: American Spinal Injury Association (ASIA) Impairment Scale. Clinical Orthopaedics and Related Research 475 (5): 1499 - 1504. DOI: 10.1007/s11999016-5133-4

4. Aoude A, Amiot LP. (2014). A comparison of the modified Tokuhashi and Tomita scores in determining prognosis for patients afflicted with spinal metastasis. Can J Surg 201457 (3): 188 - 193. DOI: $10.1503 /$ cjs.012013

5. Pernar $\mathrm{CH}$, Ebot EM, Wilson K et al. (2018). the epidemiology of prostate cancer. Cold Spring Harbor Perspectives in Medicine 8 (12): 1 - 18. DOI: https://doi.org/10.1101/ cshperspect.a030361

6. Laufer I, Rubin DG, Lis E et al. (2013). The NOMS framework: Approach to the treatment of spinal metastatic tumors. The Oncologist 18 (6): 744 - 751. https://doi. org/10.1634/theoncologist.2012-0293

7. Bydon, M., Macki, M., Abt, N. B et al (2015). Clinical and surgical outcomes after lumbar laminectomy: An analysis of 500 patients. Surgical Neurology International 6 (4): S190 - S3. DOI: 10.4103/2152-7806.156578

8. Crnalic S, Hildingsson C, Wikström et al. (2012). Outcome after surgery for metastatic spinal cord compression in 54 patients with prostate cancer. Acta Orthopaedica 83 (1): 80 - 86. DOI: https://doi.org/10.3109/17453 674.2011 .590761 
9. Cassidy J, Baker J, Lenehan B. (2018). The role of prognostic scoring systems in assessing surgical candidacy for patients with vertebral metastasis: A narrative review. Global Spine Journal 8 (6): 638 - 651. DOI: https://doi. org/10.1177/2192568217750125

10. Choi D, Crockard A, Bunger C et al. (2010). Review of metastatic spine tumour classification and indications for surgery: The consensus statement of the Global Spine Tumour Study Group. Eur Spine J 19 (2): 215 - 222. DOI: 10.1007/s00586-0091252-x
11. Gross CE, Frank RM, Hsu et al. (2015). External beam radiation therapy for orthopaedic pathology. The Journal of the American Academy of Orthopaedic Surgeons 2 3(4): 243 - 252. DOI: https://doi.org/10.5435/ JAAOS-D-14-00022 